\title{
Hemispheric differences for the integration of stimulus levels and their contents: Evidence from bilateral presentations
}

\author{
GREGOR VOLBERG and RONALD HÜBNER \\ Universität Konstanz, Konstanz, Germany
}

\begin{abstract}
Several studies have demonstrated that hemispheric differences for the processing of hierarchical letter stimuli are more likely to occur when the letters at the levels are associated with conflicting responses. Typically, a single stimulus is presented, so that the conflict occurs between the global and the local levels of the same stimulus. Our hypothesis is that in this situation, conflict resolution requires integration of the letters and their respective levels and that the hemispheres differ in this integration process. According to this integration theory, the favorable effect of response conflict on hemispheric differences should vanish if other features, such as location, can also serve for conflict resolution. This prediction was tested in the present study by simultaneously presenting an individual hierarchical stimulus to each visual field. Conflicting letters either were arranged within one stimulus or were placed in different stimuli. In the latter case, a response conflict could also be resolved by integrating letters and locations. As was expected, there were no visual field effects in these conditions. On the other hand, visual field effects showed up when the conflicting letters were located within the same stimulus. These results support the idea that the hemispheres differ in their capacity for integrating level and form.
\end{abstract}

It is often assumed that the local and global levels of hierarchically structured stimuli are more efficiently processed in the left and right cerebral hemispheres, respectively (e.g., Hübner \& Malinowski, 2002; Martin, 1979). In response time studies, this difference can be investigated by presenting hierarchical stimuli to the left visual field/right hemisphere (LVF/RH) or to the right visual field/left hemisphere (RVF/LH) and by demanding a speeded response to the form at either the global or the local level. If the hemispheres differ in the proposed direction, responses to the global level should be faster for LVF stimuli than for RVF stimuli, whereas the opposite should hold for responses to the local level. However, although such visual field (VF) effects have occurred in a number of studies (e.g., Hübner, 1998; Martin, 1979), as a whole, the empirical support for the hemispheric difference is rather weak. For instance, one article reviewing response time studies in this area reported an almost balanced number of positive and negative results (Van Kleeck, 1989). In a more recent review, negative results even outnumbered the positive findings (Yovel, Yovel, \& Levy, 2001).

This research was supported by Grant $\mathrm{Hu}$ 432/7-4 from the Deutsche Forschungsgemeinschaft to the coauthor. We thank Anja Nörenberg, Franka Glöckner, Michael J. Maier, and Steffen Walter for their help during data acquisition. We also appreciate the helpful comments of G. Yovel, L. Paquet, and L. Ward on an earlier version of the manuscript, as well as those of S. Luck, T. Carr, and two anonymous reviewers. Correspondence concerning this article should be addressed to G. Volberg, Institut für Experimentelle Psychologie, Universität Regensburg, Universitätsstr. 31, D-93053 Regensburg, Germany (e-mail: gregor.volberg@psychologie .uni-regensburg.de).
One might argue that the results from response time studies are not very reliable, because they rely on VF effects that provide only an indirect measure of hemispheric differences. If brain activity was measured by means of functional brain-imaging techniques, it often turned out that the left and the right temporo-occipital and temporo-parietal areas were activated during local and global processing, respectively (Martinez et al., 1997; Weber, Schwarz, Kneifel, Treyer, \& Buck, 2000). Also, studies with brain-damaged patients suggest that these brain regions are crucial (Delis, Robertson, \& Efron, 1986; Lamb, Robertson, \& Knight, 1990; Robertson \& Lamb, 1991). However, results obtained by more direct methods are inconclusive as well. For instance, of three studies in which positron emission tomography was used to asses brain activity during local and global processing, only one showed effects in the expected direction (Fink et al., 1996). The other two either failed to replicate this result (Heinze, Hinrichs, Scholz, Burchert, \& Mangun, 1998) or even showed a reversed effect (Fink, Marshall, Halligan, Frith, \& Frackowiak, 1997). Mixed results are also present in studies measuring event-related brain potentials (ERPs; e.g., Han, Fan, Chen, \& Zhuo, 1999; Heinze \& Münte, 1993; Johannes, Wieringa, Matzke, \& Münte, 1996) as well as in neuropsychological studies. Whereas, in some of them, lesions in the $\mathrm{LH}$ and $\mathrm{RH}$ led to a respective impairment of local and global processing (Delis et al., 1986; Lamb et al., 1990; Robertson \& Lamb, 1991), in other studies, no such effects occurred (Polster \& Rapcsak, 1994; Schatz, Craft, Koby, \& DeBaun, 2004). For example, Schatz et al. conducted a global/local task with 14 children with focal 
lesions of the LH. According to the proposed hemispheric specialization, it was expected that these patients would show poor local processing. However, the results did not differ from those for healthy controls (but see also Moses $\&$ Stiles, 2002, for a developmental study with positive results).

What could be the reason that positive outcomes have not always been observed? One possible cause has been identified by Van Kleeck (1989). In his meta-analysis of response time studies, he noticed that for incongruent stimuli (i.e., stimuli where the forms at the global and local levels are mapped onto different responses) VF effects were obtained in five out of six cases, whereas for congruent stimuli, corresponding effects occurred only in two out of five studies. This observation led Van Kleeck to hypothesize that VF effects get amplified in conflicting conditions. In line with former investigators, he assumed that the LH has a slight advantage for processing the local level, whereas the global level is processed slightly more efficiently in the RH. Importantly, this distinction applies not only to the letter at the target level, but also to that at the nontarget (distractor) level. Thus, depending on the target level and the VF of the stimulus, either the target is processed relatively strongly whereas the distractor is processed relatively poorly, or vice versa. For incongruent stimuli, this leads directly to an amplification of VF effects.

Van Kleeck's (1989) idea can best be demonstrated by means of a numerical example. Assume that the letters at the better processed level produce stronger response activations than do those at the poorer processed one (say, 12 , in comparison with 10 , units of response activation). Furthermore, it can be assumed that activations arising from letters at the unattended level are reduced by a certain factor (e.g., 0.5), in comparison with those emerging from the attended one. Now, if the task is to identify the local letter, then for incongruent stimuli, where the incorrect activation arising from the unattended level interferes with the activation of the correct response, the resulting activations for LVF and RVF stimuli are $(12-10 / 2)=$ 7 and $(10-12 / 2)=4$, respectively. By contrast, in the congruent case, the correct activation emerging from the unattended level adds up to that of the attended one, so that the respective activations for LVF and RVF stimuli are $(12+10 / 2)=17$ and $(10+12 / 2)=16$. The corresponding VF effects can be calculated by subtracting the results for stimuli presented to the "poorer" hemifield from those presented to the "better" one. Thus, for incongruent stimuli the VF effect is $7-4=3$, whereas it is $17-16=1$ for congruent ones. An analogous reasoning holds for the global target level. Therefore, response conflicts generally increase the otherwise small and noisy VF effects.

In order to test this amplifier hypothesis, Hübner and Malinowski (2002) conducted a series of experiments in which they systematically varied the amount of interference between the levels. They reasoned that if Van Kleeck's (1989) hypothesis was correct, the corresponding VF effects should vary accordingly; that is, more interference should lead to larger VF effects. However, this was not the case. Rather, it turned out that response conflicts were generally favorable for producing VF effects irrespective of their size.

By considering these results, the question arises as to how response conflicts can modulate the occurrence of hemispheric differences in a qualitative manner. To answer this question, Hübner and Malinowski (2002) proposed a binding account. They assumed that the response selection process differs for congruent and incongruent stimuli. Since for congruent stimuli, both the form at the local level and that at the global level activate the same correct response, response selection can take place relatively early and without specific level information being considered. A different situation exists for incongruent stimuli. In this case, the two forms activate different response codes. Consequently, form information alone is not sufficient for selecting the correct response. Rather, in this situation, level information also has to be taken into account. In other words, response selection for incongruent stimuli requires mental representations where level and form are integrated. Hübner and Malinowski hypothesized that the hemispheres differ in their capacity for such an integration process. The $\mathrm{LH}$ and $\mathrm{RH}$ have higher capacities for binding forms to the local and global levels of a hierarchical stimulus, respectively.

The integration account of global/local processing has recently been supported by a masking study with letters as stimuli (Hübner \& Volberg, 2005). If the theory is correct, the letters of hierarchical stimuli should be represented independently of their levels at early stages of processing - that is, before levels and letters are integrated. Consequently, if the integration process is disrupted by a mask, letter information should be available without level information. If, under such conditions, subjects have to identify the letter at a prespecified level, they should often confuse the letters at the global and local levels. This is exactly what we found (Hübner \& Volberg, 2005). Thus, our results support the idea that letters can be coded independently of their levels, which makes them similar to simple features (Treisman \& Gormican, 1988).

In the present study, further evidence is provided for the idea that the integration of levels and letters is crucial for obtaining VF effects in hierarchical processing. Our earlier results, that response conflicts favor hemispheric differences (Hübner \& Malinowski, 2002; Malinowski, Hübner, Keil, \& Gruber, 2002; Volberg \& Hübner, 2004), provide only indirect evidence for the integration account. Because in these studies response conflicts always occurred between the levels of the same stimulus, and because the integration of form and level is necessary to resolve such conflicts, one cannot distinguish whether response conflicts per se or the requirement to integrate letter and level was crucial for the VF effects. Consequently, our data are also compatible with the hypothesis that response conflicts are generally sufficient for obtaining VF effects, whatever the reason might be. Thus, in order to show that the integration hypothesis is valid, further experiments are necessary, in which the effect of response conflicts is dissociated from that of level and letter integration. A series of such experiments was conducted in the present study. 
Although our integration theory was originally based on data showing that response conflicts are favorable for VF effects, in its present form (Hübner \& Volberg, 2005), it also predicts that this relation should hold only under certain circumstances. It can be assumed that a response conflict leads to VF effects only if the interfering letters occur at the same time at the same location. Only then is it necessary to resolve the response conflict by taking level information into account. If the letters are separated in time and/or space or if they differ in other basic features, response conflict can easily be resolved by binding the letters to their location, to their time window, or to the other features. Because there is no reason to assume that the hemispheres differ with respect to integration processes other than that for level and form, no VF effects are expected in these cases.

The objective of the present study was to test the spatial aspect of the integration hypothesis. This was obtained by distributing two letters to the levels of two separate hierarchical stimuli (Paquet, 1992; Paquet \& Merikle, 1988). The remaining levels were filled with neutral forms. Both hierarchical stimuli were simultaneously presented to the LVF and the RVF, and the task was to identify the letter at the precued level of the target stimulus, which was indicated by its color. Since only two letters were present in each display, they either could occur together within the target stimulus or were distributed across target and distractor stimuli. In the latter case, the letters could be either at different levels or at the same level of the two stimuli (see Figure 1).
By considering the examples shown in Figure 1, one can see that the same-stimulus/different-levels condition is similar to the normal, single-stimulus paradigm with respect to letter distribution. As was argued above, response conflicts produced by such stimuli can be resolved only by integrating level and letter. Accordingly, we expected VF effects for this condition. However, when the letters are part of different stimuli, a response conflict can also be resolved by binding the letters to their location (or even to their color). Consequently, although reliable congruency effects were expected for the latter stimulus types, we did not predict any VF effects.

Our integration hypothesis can also be contrasted with the amplifier hypothesis (Van Kleeck, 1989). For the same-stimulus/different-levels condition, it predicts the same result as the integration approach - that is, VF effects for incongruent stimuli. Also, it does not predict VF effects for incongruent different-stimuli/different-levels stimuli. Depending on the target level and the target VF, the letters would produce either two strong or two weak activations in this condition, so that a balance of the activations would be the consequence. However, the amplifier approach also predicts VF effects for the incongruent different-stimuli/same-level condition. In this case, one weak and one strong response activation always occur, so that a reasoning analogous to that for normal hierarchical stimuli can be applied. For example, if the local level of an incongruent stimulus has to be identified, the VF effect is [12 (local/RVF) - 10/2 (local/LVF)] - [10 (local/LVF) $12 / 2($ local $/ \mathrm{RVF})]=3$. In the congruent case, it would be

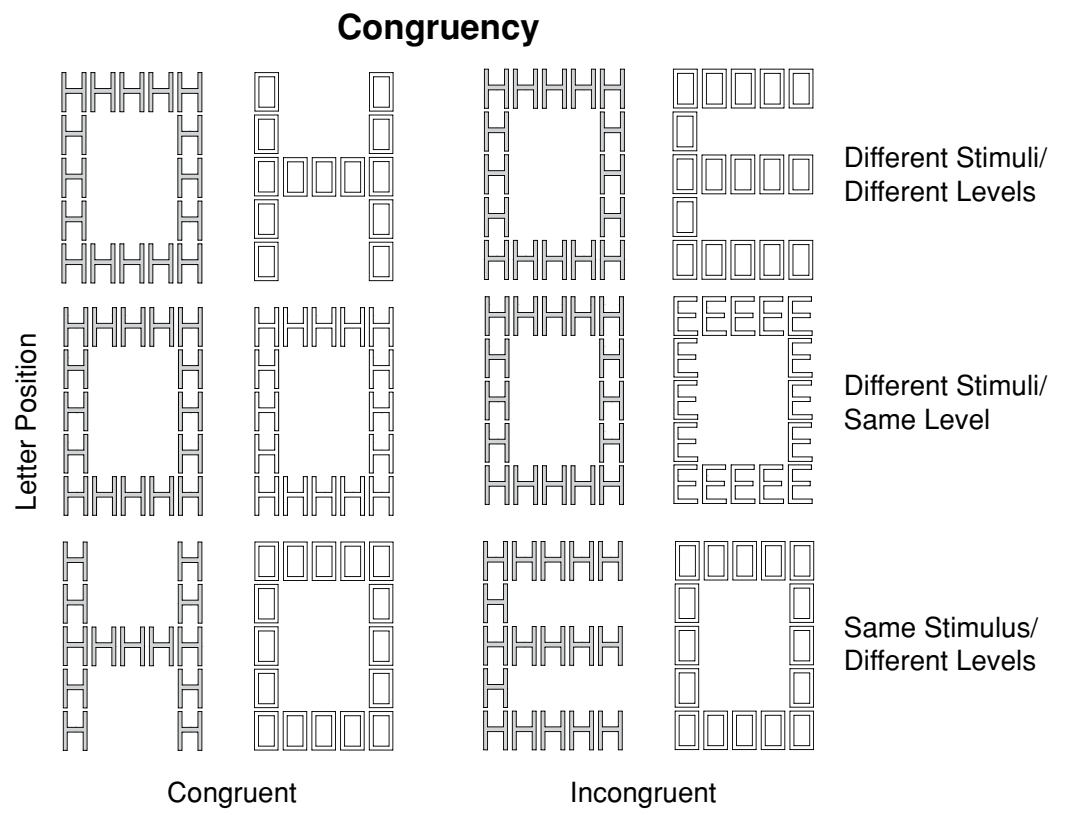

Figure 1. Typical examples of the stimulus displays used (gray = target stimulus, white $=$ distractor stimulus). Each display contained two letters, which were either congruent or incongruent. The letters could occur in one of three positions relative to each other: different-stimuli/different-levels, different-stimuli/same-level,and same-stimulus/ different-levels. The stimuli were actually drawn as white or yellow outlines on a black background. 
$[(12+10 / 2)-(10+12 / 2)]=1$. The same argument applies to situations with a global target level. Thus, the amplifier hypothesis also predicts an increased VF effect for incongruent stimuli in this condition.

\section{EXPERIMENT 1}

In the first experiment, subjects were instructed to identify the letter at a given level of an LVF or RVF stimulus, and congruent or incongruent letters were arranged either within the same stimulus or at the levels of a stimulus presented to the opposite VF. According to the integration hypothesis, VF effects should occur only in the former condition, because this is the only situation in which the integration of level and form is required for the correct response selection. On the other hand, it is possible that response conflicts generally produce VF effects. If this is the case, VF effects should occur in all three conflict conditions.

Moreover, one can again consider the predictions derived from the amplifier hypothesis. According to this approach, VF effects should occur for incongruent stimuli in the same-stimulus/different-levels and different-stimuli/ same-level conditions.

\section{Method}

\section{Subjects}

The subjects were 16 volunteers ( 13 of them female, 3 male; mean age 27.3 years), who either fulfilled course requirements or received a fee for participation. All the subjects had normal or corrected-tonormal vision and were right-handed by self-report.

\section{Apparatus}

The stimuli were produced by a personal computer and presented on a 21 -in. color monitor (Sony 500PS). The screen resolution was $1,024 \times 768$ pixels at a vertical refresh rate of $85 \mathrm{~Hz}$. Responses were given on a two-button device and were registered by the same computer as that used to control the stimulus presentation.

\section{Stimuli}

Each stimulus display contained two compound stimuli, which occurred to the left and to the right of the screen midline (Figure 1). The distance from the screen center to the inner edge of the stimuli was $0.9^{\circ}$. The global form was constructed from identical local forms in a $5 \times 5$ grid. Local and global forms subtended $0.7^{\circ} \times 0.9^{\circ}$ and $4.3^{\circ} \times 5.9^{\circ}$ of visual angle, respectively, at a viewing distance of $110 \mathrm{~cm} . \mathrm{H}$ and $\mathrm{E}$ served as letters, which were assigned to different response keys. In addition, there was a neutral form, O. Each stimulus display contained only two letters, which were either congruent ( $\mathrm{H}$ and $\mathrm{H}$ or $\mathrm{E}$ and $\mathrm{E})$ or incongruent $(\mathrm{H}$ and $\mathrm{E})$. The remaining two levels contained the neutral form. There were three different conditions with respect to the position of the letters. They could be part of two different stimuli and appear either at a different level (differentstimuli/different-levels) or at the same level (different-stimuli/samelevel). If the letters were part of the same stimulus, they could appear only at different levels (same-stimulus/different-levels). One compound stimulus was drawn in yellow, and the other in white color, both on a black background. Whether white or yellow indicated the target stimulus was balanced across subjects.

\section{Procedure}

Each trial started with a cue, which was presented for $600 \mathrm{msec}$ at the center of the screen and also served as fixation. The letters $G$ and $\mathrm{L}$ were used to indicate global and local as target level, respectively. After a blank interval of $300 \mathrm{msec}$, a stimulus display was shown for
235 msec. ${ }^{1}$ The task was to identify the letter at the target level of the target stimulus as quickly and accurately as possible by pressing an associated button with the index or middle finger of the same hand. After the response, a blank screen was shown for $1,000 \mathrm{msec}$ before the next trial began. Response hand and the assignment of letters to the buttons were balanced across subjects.

Four randomized factors were varied in the experiment: target level (global or local), target visual field (LVF or RVF), congruency (congruent or incongruent), and target/distractor relationship (different-stimuli/different-levels, different-stimuli/same-level, or same-stimulus/different-levels). The subjects performed 20 blocks of 96 trials within a single session of about $1.5 \mathrm{~h}$. The first 4 blocks were considered as practice and were excluded from further analysis, so that 64 trials per subject remained for each of the 24 conditions.

\section{Results}

Response latencies for correct responses, as well as error rates, were entered into a four-factorial ANOVA with repeated measures on all factors. Post hoc comparisons were conducted by means of planned contrasts.

\section{General Effects}

Response times. The data showed a global advantage of 22 msec. Furthermore, latencies for the differentstimuli/different-levels and different-stimuli/same-level conditions (both $554 \mathrm{msec}$ ) were shorter than those for the same-stimulus/different-levels condition (566 msec). These differences were reflected in significant effects of target level $[F(1,15)=20.09, p<.001]$ and target/ distractor relationship $[F(2,30)=6.35, p<.01]$.

Error rates. Responses were more accurate in the different-stimuli/different-levels $(3.77 \%$ errors $)$ and different-stimuli/same-level (3.20\%) conditions than in the same-stimulus/different-levels condition (6.59\%). The corresponding main effect was significant [target/distractor relationship, $F(2,30)=16.37, p<.001]$.

\section{Congruency Effects}

Response times. As can be seen in Table 1, responses were generally faster in congruent than in incongruent conditions. However, the congruency effect was larger in the same-stimulus/different-levels condition than in the different-stimuli/same-level condition and was larger in the different-stimuli/same-level condition than in the different-stimuli/different-levels condition. This outcome was reflected in a two-way interaction between congruency and target/distractor relationship $[F(2,30)=39.00$, $p<.001]$. Nevertheless, although the congruency effect differed, it was significant for all three single conditions [different-stimuli/different-levels, $F(1,15)=11.03$, $p<.01$; different-stimuli/same-level, $F(1,15)=270.57$, $p<.001$; same-stimulus/different-levels, $F(1,15)=$ $150.73, p<.001]$.

Error rates. Analogous to the response time data, responses were more accurate for congruent than for incongruent stimuli (see Table 1). This effect reached significance only in the same-stimulus/different-levels $[F(1,15)=20.27, p<.001]$ and different-stimuli/samelevel $[F(1,15)=8.85, p<.01]$ conditions. Accordingly, the interaction between congruency and target/distractor 
Table 1

Mean Response Latencies (RTs, in Milliseconds) and Error Rates (ERs, in Percentages) to Congruent and Incongruent Stimuli Conditional on the Target/Distractor Relationship in Experiment 1

\begin{tabular}{|c|c|c|c|c|c|c|}
\hline \multirow[b]{3}{*}{ Stimuli } & \multicolumn{6}{|c|}{ Target/Distractor Relationship } \\
\hline & \multicolumn{2}{|c|}{$\begin{array}{c}\text { Different } \\
\text { Stimuli/ } \\
\text { Different } \\
\text { Levels }\end{array}$} & \multicolumn{2}{|c|}{$\begin{array}{c}\text { Different } \\
\text { Stimuli/ } \\
\text { Same } \\
\text { Level }\end{array}$} & \multicolumn{2}{|c|}{$\begin{array}{c}\text { Same } \\
\text { Stimulus/ } \\
\text { Different } \\
\text { Levels }\end{array}$} \\
\hline & RT & ER & RT & ER & RT & ER \\
\hline Incongruent & 558 & 4.13 & 573 & 4.25 & 591 & 11.13 \\
\hline Congruent & 549 & 3.42 & 536 & 2.15 & 540 & 2.05 \\
\hline Incongruent - congruent & $9^{* *}$ & 0.71 & $37^{* * *}$ & $2.10^{* *}$ & $51^{* * *}$ & $9.08^{* * *}$ \\
\hline
\end{tabular}

relationships was also significant $[F(2,30)=16.76, p<$ $.001]$.

\section{VF Effects}

Response times. The VF effects, as well as the modulating effect of congruency, were as expected. Although there was an effect of congruency in all three target/distractor relationships, only in the same-stimulus/different-levels condition did it produce a VF effect. In this condition, responses to the local level were faster for target stimuli in the RVF $(587 \mathrm{msec})$ than in the LVF $(599 \mathrm{msec})$, whereas the opposite held for responses to the global level (604 vs.
$575 \mathrm{msec}$, respectively). The results can be seen in Figure 2.

The pattern of results was reflected in a significant interaction between all four factors $[F(2,30)=11.57, p<.001]$. It was decomposed by separately analyzing the different target/distractor relationships. Only for the same-stimulus/ different-levels condition did a significant three-way interaction between target level, target visual field, and congruency show up $[F(1,15)=11.61, p<.01$; different-stimuli/ different-levels, $F(1,15)=2.92, p>.10$; different-stimuli/ same-level, $F(1,15)=0.50, p>.10]$. A further decomposition of the same-stimulus/different-levels condition re-

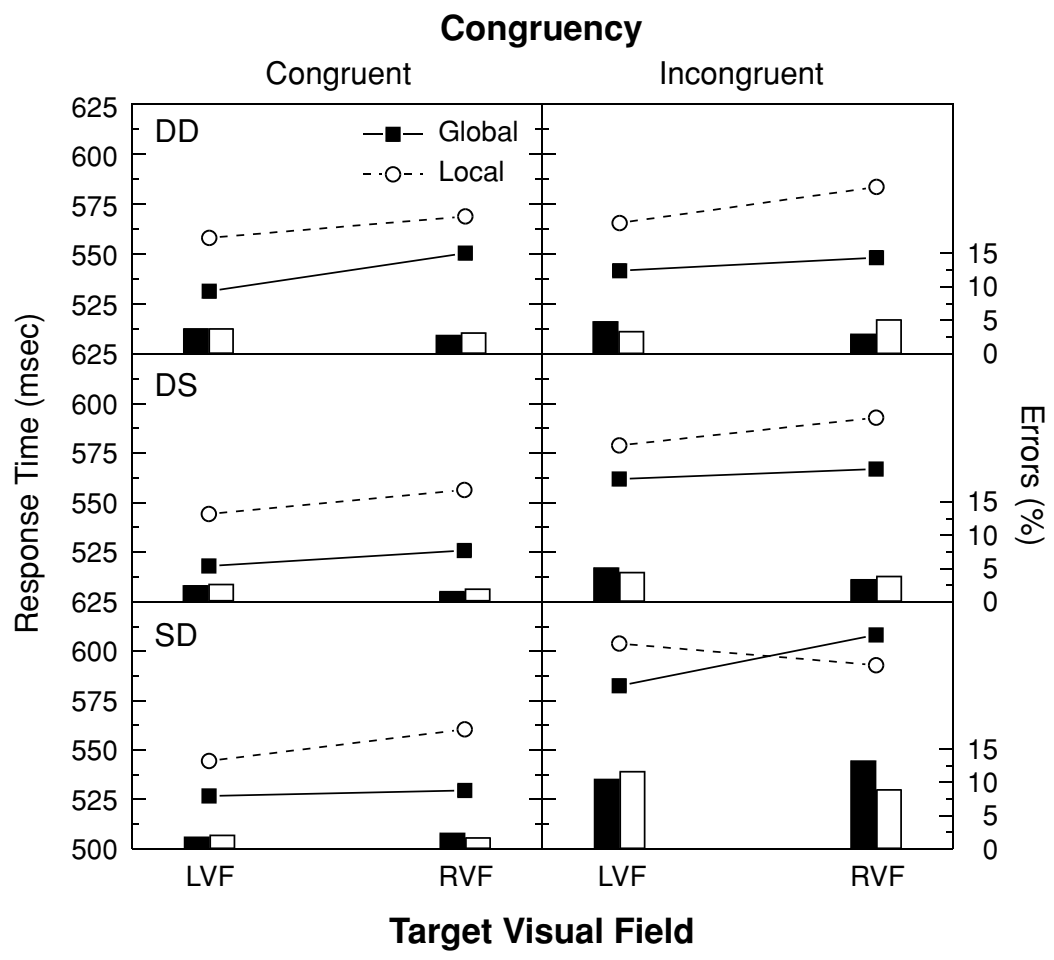

Figure 2. Mean response latencies and error rates to global and local targets as a function of the factors of target visual field, congruency, and target/distractor relationship in Experiment 1. DD, different-stimuli/different-levels; DS, different-stimuli/ same-level; SD, same-stimulus/different-levels; LVF, left visual field; RVF, right visual field. 
vealed that VF effects were reliable only for incongruent stimuli $[F(1,15)=5.55, p<.05]$.

Error rates. Similar to the response time results, congruency had a moderating effect on VF effects only in the same-stimulus/different-levels condition. In this situation, responses to the local level were more accurate for target stimuli in the RVF $(8.99 \%)$ than for those in the LVF $(11.72 \%)$, and the reversed pattern occurred for the global level (RVF, 13.28\%; LVF, 10.55\%; see Figure 2).

Consequently, the statistical analysis revealed a significant three-way interaction between target level, target visual field, and target/distractor relationship $[F(2,30)=$ $6.09, p<.01]$. It was decomposed by separately analyzing the interaction between target level and target visual field for the three target/distractor relationships. Significant results showed up only in the same-stimulus/differentlevels condition $[F(1,15)=5.04, p<.05]$. In order to see whether the results corresponded to those for the response time data, the same-stimulus/different-levels condition was further decomposed by computing separate VF effects for congruent and incongruent stimuli. They were not significant for congruent stimuli $[F(1,15)=1.07, p=.32]$ but approached significance for incongruent ones $[F(1,15)=$ $2.99, p=.10]$.

\section{Discussion}

Our aim in this experiment was to test whether response conflicts also lead to VF effects for global/local processing if the conflicting information is distributed across two spatially separated stimuli. According to the integration hypothesis, no VF effects should occur in these situations, because no integration of form and level is required for a correct response selection. Rather, VF effects were expected only in the same-stimulus/different-levels condition.

As can be seen in Figure 2, the data are in line with these predictions. First of all, they show that response conflicts are not generally favorable for hemispheric differences. Although the congruency effect was significant in all three stimulus conditions, it modulated the VF effects only in the same-stimulus/different-levels condition. Moreover, as was expected, in this condition VF effects showed up only for incongruent stimuli. It is worth noting that the obtained VF effects were of equal size or even larger than those observed in earlier studies with single stimuli (36 msec, as compared with $39 \mathrm{msec}$ in Volberg \& Hübner, 2004, or with $19 \mathrm{msec}$ in Hübner \& Malinowski, 2002, Experiment 1). Thus, the data show that bilateral displays are suitable for investigating VF effects in global and local processing (Boles, 1987).

From the amplifier hypothesis (Van Kleeck, 1989), it was derived that VF effects should occur also for incongruent different-stimuli/same-level stimuli. However, no such effects were observed. One might argue that VF effects did not show up because the induced conflict was weaker than that in the same-stimulus/different-levels condition. However, the corresponding congruency effect was nevertheless reliable. Thus, it is unlikely that such an explanation holds. By considering these findings, as well as the results of Volberg and Hübner (2004) and of Hübner and Malinowski (2002), it is obvious that the amplifier hypothesis cannot explain the effect of response conflicts on VF effects in hierarchical processing.

The data are also at odds with the hypothesis that response conflicts generally produce VF effects. However, one might argue that our test was inadequate, because the letters were not only arranged within the same stimulus or distributed across different stimuli, but also projected to the same or to different hemispheres, respectively. For example, given a target stimulus in the LVF, in the differentstimuli condition the target letter and the nontarget letter were projected to the $\mathrm{RH}$ and the $\mathrm{LH}$, respectively. In contrast, in the same-stimulus condition, both the target and the distractor letters were projected to the RH. Thus, two factors varied together: the position where the letters were presented (same vs. different stimuli) and the hemisphere where they were supposedly processed (same vs. different hemispheres). It is conceivable that response conflicts lead to hemispheric differences only if the conflicting letters are presented to the same hemisphere. Indeed, such a conjecture is supported by a study of Weissman and Banich (1999), in which they found that an interhemispheric interaction reduced interstimulus interference. Such a mechanism would explain why no VF effects occurred in the different-stimuli/ different-levels and different-stimuli/same-level conditions of this experiment. We addressed this objection in our next experiment.

\section{EXPERIMENT 2}

In this experiment, one stimulus was presented to the LVF or RVF, whereas the other was simultaneously shown in the central VF (CVF). In a small percentage of trials, the target stimulus appeared in the CVF, together with a distractor stimulus in the LVF or the RVF. However, with respect to VF effects, the relevant trials were those in which the target stimulus appeared in the LVF or the RVF, together with a distractor stimulus in the CVF. In these trials, it should be ensured that for all three types of letter distributions, the distractor was projected to the same hemisphere as the target letter. For example, in the different-stimuli/different-levels condition with a target stimulus in the LVF, the target letter, together with the CVF distractor, were projected to the $\mathrm{RH}$, and in the RVF condition, the target, together with the CVF distractor, was projected to the $\mathrm{LH}$.

The predictions were as follows. If response conflicts produce VF effects only in cases in which the letters are processed within the same hemisphere, they should occur for all three target/distractor relationships with that procedure. On the other hand, if the integration hypothesis is correct and it is crucial that target and distractor letters occur in the same stimulus, VF effects should again occur only in the same-stimulus/different-levels condition.

\section{Method}

With respect to the stimuli, apparatus, and the general procedure, the second experiment was identical to the first one. The main differ- 
Table 2

Mean Response Latencies (RTs, in Milliseconds) and Error Rates

(ERs, in Percentages) to Congruent and Incongruent Stimuli Conditional on the Target/Distractor Relationship in Experiment 2

\begin{tabular}{|c|c|c|c|c|c|c|}
\hline \multirow[b]{3}{*}{ Stimuli } & \multicolumn{6}{|c|}{ Target/Distractor Relationship } \\
\hline & \multicolumn{2}{|c|}{$\begin{array}{c}\text { Different } \\
\text { Stimuli/ } \\
\text { Different } \\
\text { Levels }\end{array}$} & \multicolumn{2}{|c|}{$\begin{array}{c}\text { Different } \\
\text { Stimuli/ } \\
\text { Same } \\
\text { Level }\end{array}$} & \multicolumn{2}{|c|}{$\begin{array}{c}\text { Same } \\
\text { Stimulus/ } \\
\text { Different } \\
\text { Levels }\end{array}$} \\
\hline & RT & ER & RT & ER & RT & ER \\
\hline Incongruent & 639 & 4.03 & 654 & 5.15 & 694 & 13.54 \\
\hline Congruent & 652 & 4.36 & 602 & 3.22 & 613 & 2.08 \\
\hline Incongruent - congruent & -13 & -0.33 & $52^{* * *}$ & $1.93^{*}$ & $81^{* * *}$ & $11.46^{* * *}$ \\
\hline
\end{tabular}

ence was the position of the two stimuli within the VF. One stimulus in the LVF or RVF was presented together with a second stimulus in the CVF, where the distance between the outer edge of the CVF stimulus and the inner edge of the LVF/RVF stimulus was $0.9^{\circ}$ of visual angle. In $75 \%$ of the trials, the target stimulus was shown in the LVF or RVF, whereas in $25 \%$ of the trials, it appeared in the CVF.

The subjects were 16 right-handed students at the Universität Konstanz ( 7 of them female, 9 male; mean age 24.9 years). After 2 training blocks, they performed 16 blocks with 96 trials each in two different sessions. Trials with target stimuli in the CVF were excluded from the analysis because they were not informative with respect to VF effects. Therefore, 48 observations per condition and subject remained.

\section{Results}

The latencies of correct responses, as well as the error rates, were subjected to a four-factorial ANOVA with repeated measures on all factors. As can be seen when the data provided in Table 2 and Figure 3 are compared with those given in Table 1 and Figure 2, respectively, the general pattern of findings was very similar to that obtained in Experiment 1 . The only remarkable difference is that in the present experiment, the response times to congruent and incongruent stimuli did not differ in the differentstimuli/different-levels condition.

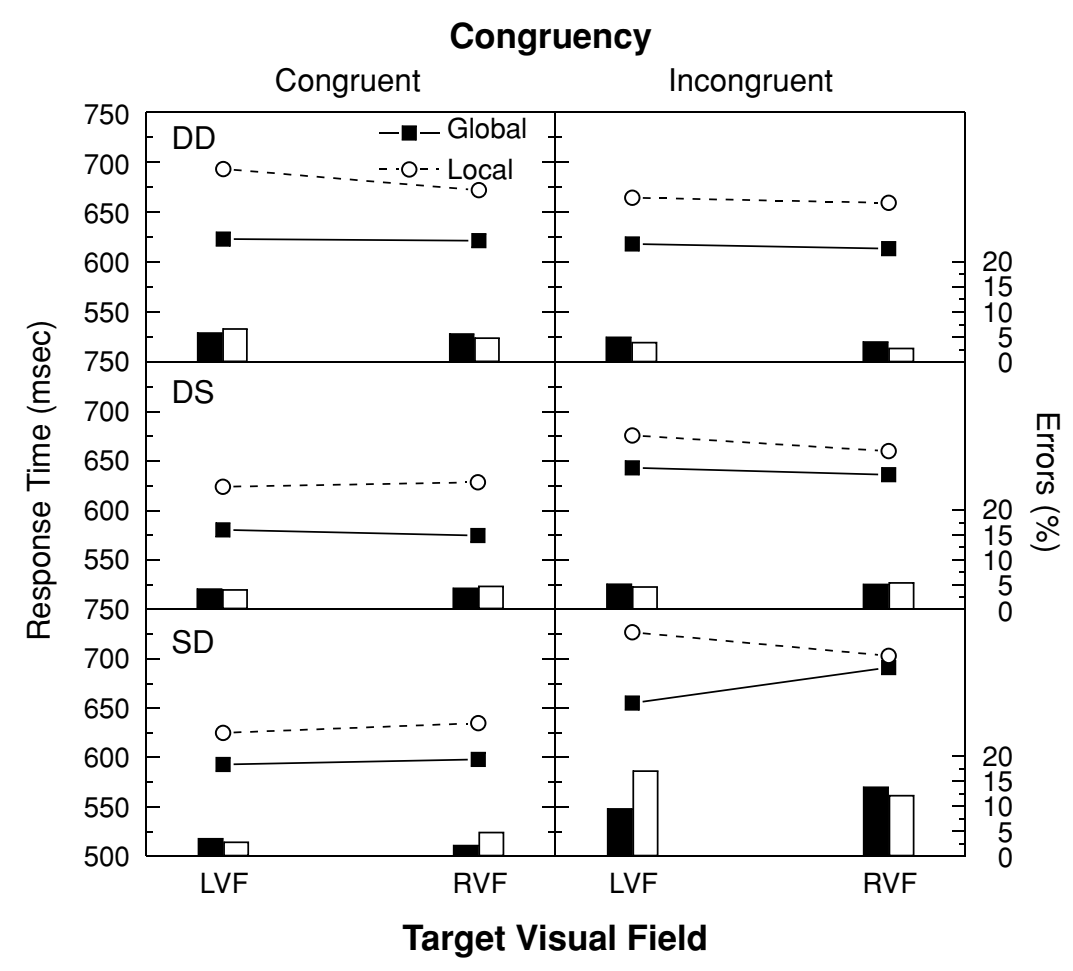

Figure 3. Mean response latencies and error rates to global and local targets as a function of the factors of target visual field, congruency, and target/distractor relationship in Experiment 2. DD, different-stimuli/different-levels; DS, different-stimuli/ same-level; SD, same-stimulus/different-levels; LVF, left visual field; RVF, right visual field. 
Most important, hemispheric differences were again restricted to the situation in which conflicting letters occurred on the levels of the same stimulus. The corresponding four-way interaction was significant [target level $\times$ visual field $\times$ congruency $\times$ target/distractor relationship: response times, $F(2,30)=3.94, p<.05$; error rates, $F(2,30)=4.47, p<.05]$. Also, the subordinate interactions were in the expected direction [target level $\times$ visual field $\times$ congruency, response times, $F(1,15)=9.42, p<$ .01 , and error rates, $F(1,15)=6.30, p<.05$; target level $\times$ visual field for incongruent stimuli, response times, $F(1,15)=8.78, p<.01$, and error rates, $F(1,15)=5.73$, $p<.05$; target level $\times$ visual field for congruent stimuli, response times, $F(1,15)=0.09, p>.70$, and error rates, $F(1,15)=0.47, p>.50]$.

\section{Discussion}

In our first experiment, the letters not only occurred within the same or in different stimuli, but also were projected to the same or to different hemispheres. Therefore, it is possible that the observed VF effects were caused by the distribution of the letters within the hemispheres, rather than by their spatial location. In order to rule out this possibility, in the present experiment, the target stimulus was shown in the LVF or the RVF, together with a distractor stimulus in the CVF. Consequently, the irrelevant letter was always projected to the same hemisphere as the relevant letter. If it was crucial for VF effects that two incongruent forms are processed within the same hemisphere, they should have appeared in all three stimulus conditions with this procedure.

This was clearly not the case. Although the congruency effect was significant in the different-stimuli/same-level and the same-stimulus/different-levels conditions, it modulated the VF effects only in the latter situation. Moreover, VF effects were again restricted to incongruent stimuli. Thus, with respect to the effect of congruency and target/ distractor relationship on VF effects, the results were the same as those in the first experiment. VF effects occurred only if there was a response conflict between the letters of the same stimulus. This can easily be explained by assuming that only in this condition was the integration of level and form necessary for resolving the conflict (Hübner \& Malinowski, 2002).

However, if we compare the same-stimulus and differentstimuli conditions, further differences besides that of the spatial arrangement of the letters were still present. The target stimuli in the different-stimuli conditions always consisted of a combination of one letter with the neutral form $\mathrm{O}$, whereas in the same-stimulus condition, the target stimuli were constructed from $\mathrm{H}$ and $\mathrm{E}$. Both of these letters feature a horizontal middle bar, and both do not have a closed outline, as does the form $\mathrm{O}$. Thus, whereas in the same-stimulus condition, the forms on the levels of the target stimulus resembled each other and, therefore, were hard to discriminate, in the different-stimuli conditions, they were dissimilar and could easily be discriminated. A further property of the different-stimuli displays was that the letters always had different colors. In contrast, in the same-stimulus condition, the letters were always drawn in the same color, which further impaired their discrimination (Briand, 1994).

There is some evidence that the ease of discriminating global and local forms is a factor for hemispheric differences. For example, in a recent ERP study, the normally observed N2 asymmetry showed up only in conditions in which the distractors changed frequently, making it harder to ignore them (Evans, Shedden, Hevenor, \& Hahn, 2000; cf. Heinze \& Münte, 1993). Likewise, in a response time study, it turned out that VF effects were greater if the saliency of the global and local levels was equal, in comparison with situations with a strong global saliency (Yovel et al., 2001). These results suggest that hemispheric differences are more likely to occur if it is hard to discriminate between the relevant and the irrelevant information. Therefore, discrimination difficulty might also be a relevant factor for the present study. Possibly, VF effects would have occurred also in the different-stimuli conditions if the discrimination of the relevant and the irrelevant letters was as hard as in the samestimulus condition.

\section{EXPERIMENT 3}

In order to test this possibility, in Experiment 3 the digit 8 was used as a placeholder instead of the O. Like the letters $\mathrm{E}$ and $\mathrm{H}$, this form has a horizontal middle bar. Therefore, the discrimination between letters and neutral forms should now be more difficult in the different-stimuli conditions. Furthermore, both of the hierarchical stimuli were drawn in white color. Which stimulus contained the target letter was defined simply by the location. It was always presented to the LVF or the RVF, whereas the distractor stimulus occurred in the CVF. Thus, in contrast to the second experiment, no CVF targets were presented. Nonetheless, since the target VF was still unpredictable, it was likely that the subjects would keep their central fixation.

Taken together, the third experiment was largely a replication of the second one, with the difference that the stimuli were modified so that the difficulty of discrimination between the target and the distractor letter was more similar between the same-stimulus and the different-stimuli conditions. Also, the predictions were the same as those in the previous experiment. If response conflicts between letters that are processed within the same hemisphere produce hemispheric differences, VF effects should occur for all three types of letter distribution. However, if the integration hypothesis is correct, VF effects should occur only in the same-stimulus situation. This prediction should hold even if the discrimination of target and distractor forms is made more difficult in the different-stimuli conditions.

\section{Method}

The apparatus and the general procedure were similar to those in the second experiment. The main difference concerned the stimuli. Both hierarchical letters were drawn in the same white foreground color, and as a neutral form, the digit 8 was used. Also, in contrast to the second experiment, the target stimulus was always presented in the LVF or the RVF, together with a distractor stimulus in the CVF. 
Table 3

Mean Response Latencies (RTs, in Milliseconds) and Error Rates

(ERs, in Percentages) to Congruent and Incongruent Stimuli Conditional on the Target/Distractor Relationship in Experiment 3

\begin{tabular}{|c|c|c|c|c|c|c|}
\hline \multirow[b]{3}{*}{ Stimuli } & \multicolumn{6}{|c|}{ Target/Distractor Relationship } \\
\hline & \multicolumn{2}{|c|}{$\begin{array}{c}\text { Different } \\
\text { Stimuli/ } \\
\text { Different } \\
\text { Levels }\end{array}$} & \multicolumn{2}{|c|}{$\begin{array}{c}\text { Different } \\
\text { Stimuli/ } \\
\text { Same } \\
\text { Level }\end{array}$} & \multicolumn{2}{|c|}{$\begin{array}{c}\text { Same } \\
\text { Stimulus/ } \\
\text { Different } \\
\text { Levels }\end{array}$} \\
\hline & RT & ER & RT & ER & RT & ER \\
\hline Incongruent & 630 & 5.10 & 640 & 6.30 & 681 & 13.11 \\
\hline Congruent & 631 & 5.23 & 564 & 3.17 & 586 & 2.32 \\
\hline Incongruent - congruent & -1 & -0.13 & $76^{* * *}$ & 3.13 & $95^{* * *}$ & $10.79^{* * *}$ \\
\hline
\end{tabular}

Sixteen students at the Universität Konstanz (12 of them female, 4 male; mean age 22.8 years) participated in the experiment. All of them were right-handed and had normal or corrected-to-normal vision. After 2 training blocks, the subjects performed 16 blocks of 96 trials within two experimental sessions. Thus, each of the 24 conditions was covered by 64 observations per subject.

\section{Results}

Response latencies of correct responses, as well as error rates, were entered into a four-factorial ANOVA with repeated measures on all factors.

Again, the general pattern of results was very similar to those obtained in Experiments 1 and 2 (see Table 3 and
Figure 4). One difference from Experiment 2 was that the congruency effect in the different-stimuli/same-level condition was significant only for the response times, but not for error rates. With respect to VF effects, the same pattern as those in both of the former experiments was observed. VF effects occurred only in the incongruent same-stimulus/different-levels condition. The four-way interaction between target level, visual field, congruency, and target/distractor relationships was significant [response times, $F(2,30)=11.86, p<.001$; error rates, $F(2,30)=6.98, p<.01]$, and the subordinate interactions in the same-stimulus/different-levels condition were as predicted [target level $\times$ visual field $\times$ congruency, re-

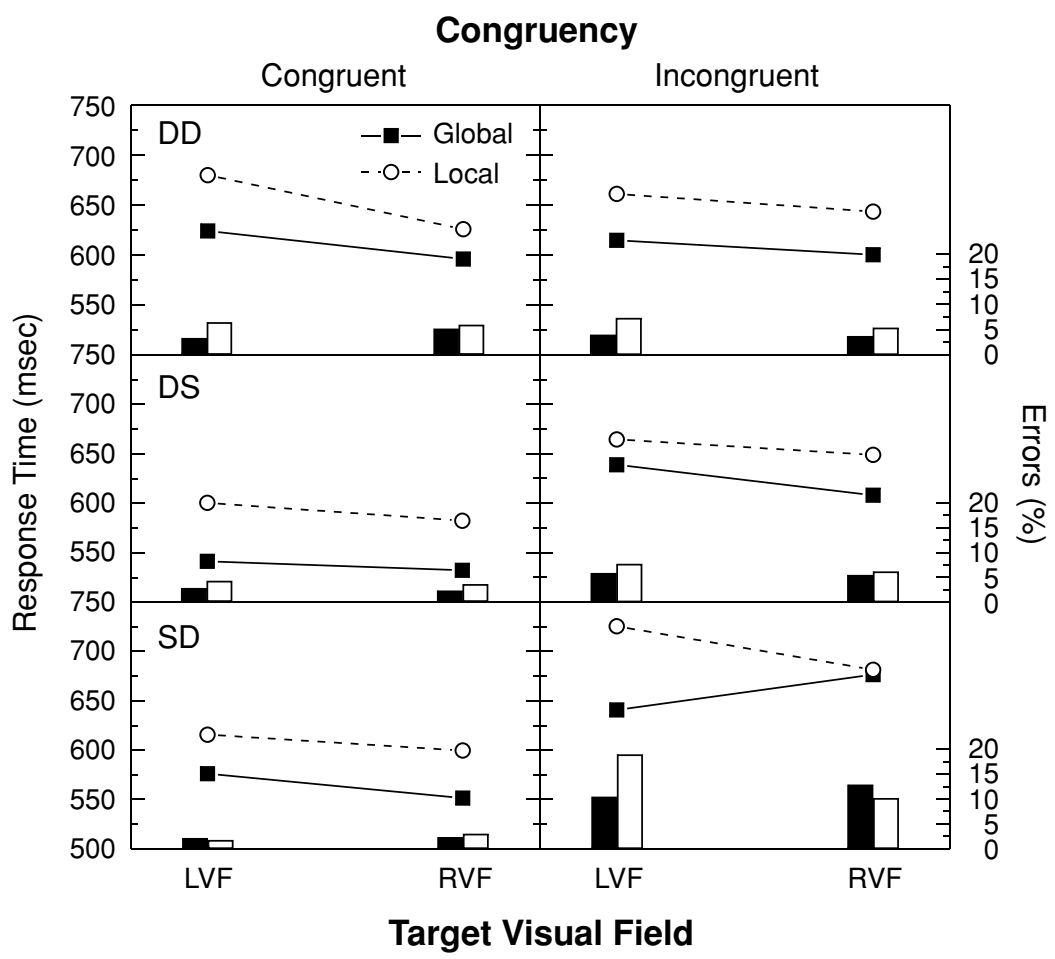

Figure 4. Mean response latencies and error rates to global and local targets as a function of the factors of target visual field, congruency, and target/distractor relationship in Experiment 3. DD, different-stimuli/different-levels; DS, different-stimuli/ same-level; SD, same-stimulus/different-levels; LVF, left visual field; RVF, right visual field. 
sponse times, $F(1,15)=19.27, p<.001$, and error rates, $F(1,15)=8.18, p<.05$; target level $\times$ visual field for incongruent stimuli, response times, $F(1,15)=11.62$, $p<.01$, and error rates, $F(1,15)=7.34, p<.05$; target level $\times$ visual field for congruent stimuli, response times, $F(1,15)=0.27, p>.60$, and error rates, $F(1,15)=1.16$, $p=.30]$.

\section{Discussion}

The present experiment was similar to the previous one, except for two changes with respect to the stimuli. Here, both of the hierarchical stimuli were drawn in white color, and 8 was used as a neutral form instead of O. Both changes served to make the stimuli in the different-stimuli conditions more similar to that in the same-stimulus condition, so that the discrimination of the relevant from the irrelevant letter was of comparable difficulty in both cases. The question was whether, under these conditions, VF effects would also show up in the conflicting different-stimuli conditions or whether they would again be restricted to cases in which the letters occurred within the same hierarchical stimulus.

The first important result is that the congruency effect in the different-stimuli/same-level condition was substantially increased, in comparison with Experiment 2. Whereas in the previous experiment, the response time and error rate differences between congruent and incongruent stimuli were $52 \mathrm{msec}$ and $1.93 \%$, respectively, they mounted to $76 \mathrm{msec}$ and $3.13 \%$, respectively, in the present experiment. This shows that the modified stimuli had the intended effect. In comparison with the second experiment, it was harder to ignore the irrelevant letter. But did this lead to a different pattern of VF effects?

The data show a clear picture. With respect to the modulating effect of congruency and of target/distractor relationship on VF effects, the results exactly mirror those in the former experiments. VF effects again occurred only if conflicting information was contained at the levels of the same stimulus. In contrast, in the two different-stimuli conditions, there was no indication of a positive effect of congruency on hemispheric differences. This was true even though the forms presented within the target stimulus were much harder to discriminate than those in Experiments 1 and 2. Thus, these results again support our hypothesis that response conflicts favor VF effects only if level and form have to be integrated for response selection.

\section{GENERAL DISCUSSION}

The starting point of this study was the observation that VF effects for the processing of hierarchical stimuli occurred mainly when the information at the levels produced a response conflict. Corresponding results have been reported in response time experiments (Hübner \& Malinowski, 2002; Van Kleeck, 1989), as well as in ERP studies (Malinowski et al., 2002; Volberg \& Hübner, 2004). However, although there is growing evidence that response conflicts are important, the underlying mechanisms are still unclear.
A simple explanation would be the amplifier hypothesis (Van Kleeck, 1989), which assumes that the different relative strengths of the levels in the two hemispheres increases the corresponding VF effects. However, as has been shown, the amplifier hypothesis cannot explain the available data (cf. Hübner \& Malinowski, 2002). Therefore, the integration hypothesis of global/local processing has been proposed as an alternative explanation (Hübner \& Malinowski, 2002; Hübner \& Volberg, 2005). According to this approach, response conflicts lead to VF effects because the resolution of the conflict requires the integration of form and level and the hemispheres differ with respect to this integration process. Contrary to the amplifier hypothesis, the integration hypothesis predicts that the favorable effect of response conflict on VF effects occurs only if level is the only feature that can be used for conflict resolution. For instance, if location can also be used, conflict should have no effect on the VF effects.

This prediction was tested in the present series of experiments by distributing conflicting letters either within the same stimulus or across two spatially separated stimuli. If the integration of letter and level is crucial for VF effects to occur, the latter stimuli should not produce any VF effects, because in this case a response conflict can more easily be resolved by binding the letters to their respective location. As our results in all three experiments show, this was indeed the case. VF effects occurred only when the conflicting letters were contained in the same stimulus (same-stimulus/different-levels). This indicates that VF effects are not directly caused by response conflicts. Rather, they occur as a result of binding mechanisms by which a response conflict is resolved (cf. Hübner \& Malinowski, 2002; Hübner \& Volberg, 2005).

On the other hand, the amplifier hypothesis (Van Kleeck, 1989 ) predicts VF effects not only for incongruent samestimulus/different-levels stimuli, but also for the incongruent different-stimuli/same-level condition. However, although significant congruency effects were also present in the latter condition, they produced VF effects only in the former condition. Therefore, the amplifier hypothesis can again be rejected as an explanation for the favorable effect of response conflicts on VF effects (see also Hübner \& Malinowski, 2002; Volberg \& Hübner, 2004).

Proponents of the amplifier hypothesis could argue that VF effects are possibly amplified only if the corresponding congruency effect exceeds a certain threshold. Perhaps there was no VF effect for different-stimuli/same-levels stimuli because, in this situation, congruency was below threshold. However, the threshold argument is not part of the amplifier hypothesis, nor can it easily be derived from it. Quite the opposite, the amplifier hypothesis actually predicts that VF effects increase proportionally with the amount of congruency (cf. Hübner \& Malinowski, 2002). Therefore, a qualitative relationship between congruency and VF effects provides more support for the integration theory than for the amplifier hypothesis. Moreover, some aspects of our present data are even at odds with the threshold idea. Although the same-stimulus/differentlevels stimuli produced the largest congruency effects 
within each experiment, this does not hold for a comparison across experiments. The congruency effect for the same-stimulus/different-levels condition in the first experiment, for which VF effects were observed, was substantially smaller than the congruency effect in the differentstimuli/same-level condition in the third experiment (51 vs. $76 \mathrm{msec}$ ), for which no VF effects occurred.

These considerations and facts strongly argue against the amplifier hypothesis and its threshold variant. Nevertheless, it is also true that within each experiment, the condition for which VF effects were observed (the samestimulus/different-levels condition) coincides with the condition that produced the largest congruency effects. Thus, some suspicion might remain that congruency of a certain degree alone could be responsible for the VF effects, rather than the combined effect of congruency and the appearance of the two letters at the same location. Although it is highly unlikely that such an account holds, it cannot definitively be ruled out by the present data. However, in comparison with the integration hypothesis, such a mechanism would be not only rather complex, but also relatively unspecified. For explaining the present results, the additional vague assumption has to be made that the amount of congruency must exceed a certain threshold that varies from experiment to experiment. In contrast, the integration hypothesis offers not only a specific mechanism, but also a parsimonious explanation of the present pattern of results.

Further evidence against the idea that congruency alone is generally sufficient for enhancing VF effects comes from a recent study with more naturalistic hierarchical objects (Hübner \& Studer, 2006). Instead of letters, the stimuli in this study consisted of a shape of an animal (global level) covered with a texture of lines or dots (local level). As a result, although the interference of shape and texture produced substantial congruency effects, it did not affect the VF effects. Rather, reliable VF effects of similar size were observed for congruent as well as for incongruent stimuli.

These results suggest that hierarchical stimuli composed of letters are special. Presumably, it is a specific property of our highly learned letters that their mental representation can be accessed for response selection without also accessing level information. Level information is additionally required only in the case of a response conflict. This explains the modulating effect of congruency on VF effects for hierarchical letters. For more naturalistic objects, it seems that content and levels are automatically integrated, so that they cannot be accessed independently. Consequently, VF effects show up irrespective of congruency.

Altogether, our pattern of results is highly compatible with the integration hypothesis of global/local processing and corresponding hemispheric asymmetries. It supports the idea that the hemispheres differ in their capacity for binding forms to their respective level. The RH and $\mathrm{LH}$ are better at integrating the global and the local levels, respectively, with their contents. The idea that form and level have to be integrated at some stage of processing presupposes that these components are separated at earlier stages of processing. This suggests that stimulus levels are similar to features or abstract categories (Treisman \& Gormican, 1988). Evidence for this idea is also provided by level repetition experiments, which show that stimulus levels are activated independently of their content and that the corresponding priming effects are of similar size for the levels (e.g., Hübner, 2000; Lamb \& Yund, 1996; Robertson, 1996; Robertson, Egly, Lamb, \& Kerth, 1993; Ward, 1982).

Furthermore, the present result, that the congruency effects between stimuli were higher when the letters occurred at the same level than when they appeared at different levels, also supports a categorical interpretation. Corresponding findings have also been reported in earlier studies (Briand, 1994; Paquet, 1992; Paquet \& Merikle, 1988). Paquet explained this result by assuming that stimulus levels are categories that help to differentiate targets from distractors. Therefore, if a distractor appears at the same level as the target, its separation is harder than when it occurs at a different level. Such an interpretation is in line with our integration hypothesis.

\section{REFERENCES}

BoLEs, D. B. (1987). Reaction time asymmetry through bilateral versus unilateral stimulus presentation. Brain \& Cognition, 6, 321-333.

BRIAND, K. A. (1994). Selective attention to global and local structure of objects: Alternative measures of nontarget processing. Perception \& Psychophysics, 55, 562-574.

Delis, D. C., Robertson, L. C., \& Efron, R. (1986). Hemispheric specialization of memory for visual hierarchical stimuli. Neuropsychologia, 24, 205-214.

Evans, M. A., Shedden, J. M., Hevenor, S. J., \& Hahn, M. C. (2000). The effect of variability of unattended information on global and local processing: Evidence for lateralization at early stages of processing. Neuropsychologia, 38, 225-239.

Fink, G. R., Halligan, P. W., Marshall, J. C., Frith, C. D., FrackOWIAK, R. S. J., \& Dolan, R. J. (1996). Where in the brain does visual attention select the forest and the trees? Nature, 382, 626-628.

Fink, G. R., Marshall, J. C., Halligan, P. W., Frith, C. D., \& FrackOWIAK, R. S. J. (1997). Hemispheric specialization for global and local processing: The effect of stimulus category. Proceedings of the Royal Society of London: Series B, 264, 487-494.

Han, S., FAN, S., Chen, L., \& Zhuo, Y. (1999). Modulation of brain activities by hierarchical processing: A high-density ERP study. Brain Topography, 11, 171-183.

Heinze, H. J., Hinrichs, H., Scholz, M., Burchert, W., \& ManGUN, G. R. (1998). Neural mechanisms of global and local processing: A combined PET and ERP study. Journal of Cognitive Neuroscience, 10, 485-498.

Heinze, H. J., \& Münte, T. F. (1993). Electrophysiological correlates of hierarchical stimulus processing: Dissociation between onset and later stages of global and local target processing. Neuropsychologia, 31, 841-852.

HÜBNER, R. (1998). Hemispheric differences in global/local processing revealed by same-different judgements. Visual Cognition, 5, 457-478.

HüBNER, R. (2000). Attention shifting between global and local target levels: The persistence of level-repetition effects. Visual Cognition, 7, 465-484.

HÜBNER, R., \& MALINOWSKI, P. (2002). The effect of response competition on functional hemispheric asymmetries for global/local processing. Perception \& Psychophysics, 64, 1290-1300.

HüBNER, R., \& STUDER, T. (2006). Hemispheric differences for global/ local processing of naturalistic objects reveal that hierarchical letter stimuli are special. Unpublished manuscript.

HüBNER, R., \& VOLBERG, G. (2005). The integration of object levels and their content: A theory of global/local processing and related hemi- 
spheric differences. Journal of Experimental Psychology: Human Perception \& Performance, 31, 520-541.

Johannes, S., Wieringa, B. M., Matzke, M., \& Münte, T. F. (1996). Hierarchical visual stimuli: Electrophysiological evidence for separate left hemispheric global and local processing mechanisms in humans. Neuroscience Letters, 210, 111-114.

Lamb, M. R., Robertson, L. C., \& Knight, R. T. (1990). Component mechanisms underlying the processing of hierarchically organized patterns: Inferences from patients with unilateral cortical lesions. Journal of Experimental Psychology: Learning, Memory, \& Cognition, 16, 471-483.

Lamb, M. R., \& Yund, E. W. (1996). Spatial frequency and attention: Effects of level-, target-, and location-repetition on the processing of global and local forms. Perception \& Psychophysics, 58, 363-373.

Malinowski, P., Hübner, R., KeIl, A., \& Gruber, T. (2002). The influence of response competition on cerebral asymmetries for processing hierarchical stimuli revealed by ERP recordings. Experimental Brain Research, 144, 136-139.

Martin, M. (1979). Hemispheric specialization for global and local processing. Neuropsychologia, 17, 33-40.

Martinez, A., Moses, P., Frank, L., Buxton, R., Wong, E., \& Stiles, L. (1997). Hemispheric asymmetries in global and local processing: Evidence from fMRI. NeuroReport, 8, 1685-1689.

Moses, P., \& Stiles, J. (2002). The lesion methodology: Contrasting views from adult and child studies. Developmental Psychobiology, 40, 266-277.

PAQUeT, L. (1992). Global and local processing in nonattended objects: A failure to induce local processing dominance. Journal of Experimental Psychology: Human Perception \& Performance, 18, 512-529.

Paquet, L., \& Merikle, P. M. (1988). Global precedence in attended and nonattended objects. Journal of Experimental Psychology: Human Perception \& Performance, 14, 89-100.

Polster, M. R., \& RAPCSAK, S. Z. (1994). Hierarchical stimuli and hemispheric specialization: Two case studies. Cortex, 30, 487-497.

ROBERTSON, L. C. (1996). Attentional persistence for features of hierarchical patterns. Journal of Experimental Psychology: General, 125, 227-249.

Robertson, L. C., Egly, R., Lamb, M. R., \& Kerth, L. (1993). Spatial attention and cuing to global and local levels of hierarchical structure. Journal of Experimental Psychology: Human Perception \& Performance, 19, 471-487.
RoberTson, L. C., \& LAMB, M. R. (1991). Neuropsychological contributions to theories of part/whole organization. Cognitive Psychology, 23, 299-330.

Schatz, J., Craft, S., Koby, M., \& DeBaun, M. R. (2004). Asymmetries in visual-spatial processing following childhood stroke. Neuropsychology, 18, 340-352.

Treisman, A., \& Gormican, S. (1988). Feature analysis in early vision: Evidence from search asymmetries. Psychological Review, 95, 15-48.

VAN KLEECK, M. H. (1989). Hemispheric differences in global versus local processing of hierarchical visual stimuli by normal subjects: New data and a meta-analysis of previous studies. Neuropsychologia, 27, 1165-1178

VolberG, G., \& HübNer, R. (2004). On the role of response conflicts and stimulus position for hemispheric differences in global/local processing: An ERP study. Neuropsychologia, 42, 1805-1813.

WARD, L. M. (1982). Determinants of attention to local and global features of visual forms. Journal of Experimental Psychology: Human Perception \& Performance, $8,562-581$.

Weber, B., Schwarz, U., Kneifel, S., Treyer, V., \& Buck, A. (2000). Hierarchical visual processing is dependent on the oculomotor system. NeuroReport, 11, 241-247.

WeIsSMAn, D. H., \& BANICH, M. T. (1999). Global-local interference modulated by communication between the hemispheres. Journal of Experimental Psychology: General, 128, 283-308.

Yovel, G., Yovel, I., \& LEVY, J. (2001). Hemispheric asymmetries for global and local visual perception: Effects of stimulus and task factors. Journal of Experimental Psychology: Human Perception \& Performance, 27, 1369-1385.

\section{NOTE}

1. The duration of $235 \mathrm{msec}$ is, in principle, enough time to perform eye movements toward the target stimulus, making it available to both hemispheres. However, because clear VF effects were obtained in all experiments, we can be sure that the subject did not carry out eye movements and that, therefore, the VF procedure worked as intended.

(Manuscript received October 13, 2005; revision accepted for publication January 10, 2006.) 